\title{
Alunos do Ensino Médio da rede pública de Fortaleza-CE e o interesse pela Botânica
}

\author{
High school students from public schools in Fortaleza-CE and the interest in Botany \\ Estudiantes de secundaria de escuelas públicas de Fortaleza-CE y el interés por la Botánica
}

\section{Resumo}

A Botânica estuda e classifica os vegetais considerando as formas, estruturas e composição; seu conhecimento está intimamente associado ao cotidiano das pessoas. Contudo, sua presença quase sempre é ignorada, de modo que, na Educação Básica, é vista como um conteúdo muito teórico, descritiva e desestimulante. Por isso, é importante saber a opinião do educando em relação à matéria e tentar promover melhorias nesta área, desta forma, objetivou-se averiguar o interesse em Botânica de alunos do Ensino Médio de duas escolas da rede pública de Fortaleza. Para tanto, foi aplicado um questionário a 71 alunos do $2^{\circ}$ ano do Ensino Médio, de duas escolas da Rede Pública Estadual de Fortaleza, CE, composto de sete perguntas, indagando-os sobre as dificuldades encontradas por eles, a forma que o professor compartilha o conteúdo, sugestões para melhorar o entendimento na disciplina, dentre outras; também participaram da pesquisa os professores de Biologia das respectivas turmas pesquisadas, para saber sua opinião sobre o assunto e sua atuação em sala de aula. Verificou-se que a maioria dos alunos tem interesse por Botânica e acham relevante conhecer sobre o assunto, contudo apresentam algumas dificuldades, especialmente quanto à nomenclatura, de modo que o docente apresenta papel fundamental para facilitar o entendimento deste conteúdo.

Palavras-chave: Biologia; Ensino de botânica; Educação básica.

\begin{abstract}
:
Botany studies and classifies plants considering their forms, structures, and composition; its knowledge is closely associated with people's daily lives. However, its presence is almost always ignored, so that, in Basic Education, it is seen as a very theoretical, descriptive, and discouraging content. Therefore, it is important to know the student's opinion about the subject and try to promote improvements in this area. Thus, this study aimed to investigate the interest in Botany of high school students from two public schools in Fortaleza. To this end, a questionnaire was applied to $712 \mathrm{nd}$ year high school students from two public high schools in Fortaleza, composed of seven questions, asking them about the difficulties they encounter, the way their teachers share the content, suggestions to improve their understanding of the subject, among others. The Biology teachers of the respective classes also participated in the research, to find out their opinion on the subject and their performance in the classroom. It was verified that most students are interested in
\end{abstract}


Botany and find it relevant to know about the subject, however, they present some difficulties, especially regarding nomenclature, so that the teacher has a fundamental role in facilitating the understanding of this content.

Keywords: Biology; Botany teaching; Basic education.

\section{Resumen:}

La Botánica estudia y clasifica las plantas teniendo en cuenta sus formas, estructuras y composición; sus conocimientos están estrechamente relacionados con la vida cotidiana de las personas. Sin embargo, su presencia es casi siempre ignorada, por lo que, en la Educación Básica, se ve como un contenido muy teórico, descriptivo y desalentador. Por lo tanto, es importante conocer la opinión del estudiante en relación con el tema y tratar de promover mejoras en esta área, por lo tanto, se pretendió investigar el interés en la Botánica de los estudiantes de secundaria de dos escuelas públicas en Fortaleza. Para ello, se aplicó un cuestionario a 71 alumnos de $2^{\circ}$ de Bachillerato, de dos escuelas de la Red Pública Estatal de Fortaleza, CE, compuesto por siete preguntas, preguntándoles sobre las dificultades encontradas por ellos, la forma en que el profesor comparte el contenido, sugerencias para mejorar la comprensión en la asignatura, entre otras; también participaron en la investigación los profesores de Biología de las respectivas clases investigadas, para conocer su opinión sobre la asignatura y su actuación en el aula. Se encontró que la mayoría de los estudiantes están interesados en la Botánica y les parece relevante conocer sobre el tema, sin embargo presentan algunas dificultades, especialmente en lo que respecta a la nomenclatura, por lo que el profesor tiene un papel clave para facilitar la comprensión de este contenido.

Palabras clave: Biología; Ensenãnza de la botánica; Educación básica.

\section{Introdução}

A Biologia faz parte das Ciências que estuda a Natureza (Ferretti, 2016), de modo que aprender seus conteúdos na Educação Básica permite maior compreensão sobre os seres vivos, em escala micro ou macroscópica (Brasil, 2006a). No Ensino Médio, ela aborda diferentes temáticas as quais podemos citar: Zoologia, Evolução, Ecologia, Citologia, Genética e Botânica (Brasil, 2006b). Este último compreende a área da Biologia que estuda os vegetais e classifica-os de acordo com suas características morfológicas e fisiológicas, reunindo-os em grupos de acordo com as suas particularidades (Minhoto, 2002).

O ensino da Botânica se faz relevante, pois as plantas são indispensáveis para a sobrevivência dos seres vivos e estão intimamente relacionadas com a vida do ser homem, como discutido por Salatino e Buckeridge (2016). Elas fornecem oxigênio, fibras para tecido, alimento, madeira, combustível (etanol), além de seus usos farmacológicos e ao longo do tempo essa relação vem se desenvolvendo, descobrindo-se novas maneiras de aproveitamento (Ferreira, Furlan \& Motta, 2012).

Apesar da relevância do ensino de Botânica, ainda há dissociação entre esta ciência e seu ensino na Educação Básica. A bibliografia especializada aponta que este conteúdo é muito teórico, desmotivador e desvalorizado no ensino de ciências (Kinoshita et al., 2006), sendo descrito pelos alunos, e até por alguns professores, como difícil, enfadonho e distante do cotidiano (Ursi et al., 2018). Por isto essa temática é frequentemente abordada de forma superficial e com o uso de métodos didáticos tradicionais que mantêm o assunto distante da realidade dos alunos (Chassot, 2003; Camargo-Oliveira, 2007; Machado \& Amaral, 2015; Silva, Almeida Jr \& Valle, 2020), sendo importante salientar que esses obstáculos não são exclusivos da Educação Básica brasileira, pois também são relatados por estudos em outros países (Hershey, 1996; Silva, Guimarães \& Sano, 2016).

Entre os diversos obstáculos do ensino de Botânica na Educação Básica brasileira, a falta de atividades práticas, uso reduzido de tecnologias, unidos à abordagem tradicional como única metodologia de ensino, são apontados como as maiores causas de desinteresse pelos alunos (Ursi et al., 2018). Desta forma é cada vez mais comum a busca por ferramentas didáticas e métodos de ensino inovadores (e.g. Nascimento et al., 2013; Edson-Chaves et al., 2015; Paz \& Souza, 2016; Nascimento et al., 2017; Lemos et al., 2018; Silva et al., 2019; Costa, Duarte \& Gama, 2019; Dias et al., 2020; Marinho \& Valle, 2020; BrasilPeixoto et al., 2021), onde o professor desenvolva o pensamento crítico e se colocar no papel de mediador na construção do conhecimento pelo aluno, possibilitando uma aprendizagem significativa (Vasques et al., 2021a). Por entender essas demandas e se deparar frequentemente com os obstáculos associados ao ensino de Botânica Vasques et al. (2021b) reuniram temáticas 
importantes para discutir, compreender e desmistificar o ensino de Botânica no Brasil, norteando perspectivas e práticas relacionadas ao Aprendizado Ativo deste conteúdo.

Diante disso, objetivou-se averiguar o interesse em Botânica de alunos do Ensino Médio da rede pública de Fortaleza, visando à melhoria da prática pedagógica. Para isso, é fundamental conhecer a maneira como o conteúdo é abordado em sala de aula, verificar as dificuldades dos alunos no aprendizado de Botânica, e então, sugerir possíveis mudanças didáticas da disciplina.

\section{Metodologia}

A pesquisa tem caráter descritivo e qualitativo com a intenção de obter a opinião do público-alvo, alunos e professores de escolas públicas da rede estadual de ensino de Fortaleza. Para Gil (1999; 2002), este tipo de pesquisa tem como essência descrever as características de certo grupo ou fenômeno ou estabelecimento de relações entre variáveis, ao mesmo tempo em que, dado a natureza da pesquisa tenha procurado adentrar-se na subjetividade dos indivíduos observados, oferecendo-lhe maior liberdade para expressão.

Para a realização da pesquisa, foram selecionadas duas escolas públicas de Fortaleza, CE. A escola EJMS é uma escola de tempo integral com aulas regulares e cursos profissionalizantes, enquanto a escola EHCS ainda atendia turmas de $9^{\circ}$ ano do Ensino Fundamental e as três séries do Ensino Médio. Vale ressaltar que ambas as instituições possuem laboratórios multidisciplinares de Ciências e todos seus alunos dispunham de livro didático. Por questões éticas preferimos utilizar acrônimos para as duas instituições de ensino. Um professor de cada escola, responsável pelas turmas, foi escolhido para participar do estudo. Os docentes entrevistados responderam um questionário composto por cinco questões discursivas voluntariamente (Quadro 1). Posteriormente teve seu discurso analisado e confrontado com as respostas dos alunos.

Quadro 1. Questões aplicadas no questionário referente aos docentes.

\begin{tabular}{ll}
\hline Questões aplicadas aos docentes \\
$\begin{array}{ll}\text { 1) Qual a sua formação? } & \text { 4) Quais metodologias utilizadas para ministrar Botânica? } \\
\text { 2) Você gosta de lecionar? } & \text { 5) Você contextualiza a Botânica com outros assuntos? } \\
\text { 3) Você gosta de Botânica? } & \end{array}$
\end{tabular}

Fonte: Autores.

Quanto aos discentes, o público-alvo foi composto por 71 alunos (46 alunos eram da EHCS e 25 alunos da EJMS 59,91\% do universo amostral) do $2^{\circ}$ ano do Ensino Médio, pois, nestas instituições, o componente curricular de Botânica era oferecido nesta etapa. Os participantes tinham idade entre 16 e 18 anos, de ambos os sexos, os quais receberam esclarecimentos sobre o estudo e responderam ao questionário de seis questões discursivas (Quadro 2), que tinham como objetivo averiguar a opinião dos estudantes sobre Botânica, seus conhecimentos prévios, dificuldades, impressões e sugestões sobre a disciplina. Os dados foram analisados quantitativamente e apresentados por meio de gráficos. 
Quadro 2. Questões aplicadas no questionário referente aos discentes.

\begin{tabular}{|ll|}
\hline Questões aplicadas aos discentes & \\
\hline 1) Você gosta de Botânica? Por quê? & 5) Como o professor aborda o conteúdo em sala de \\
2) Você acha relevante conhecer Botânica? & aula? \\
3) Qual a importância da Botânica em seu cotidiano? & 6) Você possui alguma sugestão para melhorar seu \\
4) Você tem alguma dificuldade nessa disciplina? Qual seria? & entendimento no assunto? \\
\hline
\end{tabular}

Fonte: Autores.

A pesquisa foi realizada com o consentimento dos respondentes e assinatura do um Termo de Consentimento Livre e Esclarecimento assinado pelos docentes, alunos e responsáveis dos discentes.

\section{Resultados e Discussão}

\subsection{Sobre os professores}

De acordo com as respostas fornecidas pelos docentes ambos são licenciados em Ciências Biológicas, sentem satisfação em ensinar, porém somente um se identificava com o conteúdo de Botânica. Estudos que abordam o ensino de Botânica em escolas do Ensino Fundamental e Ensino Médio correlacionam à dificuldade dos professores de se identificar com temas de Botânica com lacunas ou deficiências na formação do mesmo (Silva, Cavallet \& Alquini, 2006). Este fator é determinante para a socialização das informações e limitador para o processo de ensino-aprendizagem (Pes, 2019). No contexto educacional, a falta de interesse demonstrada por parte do docente reflete diretamente no interesse dos alunos, que também passam a não entenderem a relevância do assunto ministrado, tornando-os indiferentes à disciplina estudada (Costa et al., 2019). Sabendo disso, é necessário que o docente atuante em sala de aula procure participar de formações continuadas a fim de preencher as lacunas deixadas pelos cursos de graduação (Pes, 2019).

Quanto às metodologias utilizadas pelos docentes, ambos comentaram que ministram aulas por meio da exposição de conteúdo com slides e eventualmente utilizam documentários, ou realizam aulas práticas no laboratório da escola. Além do mais, os entrevistados costumam contextualizar a matéria com outras disciplinas, principalmente com ecologia. Isso mostra o comprometimento dos professores em aperfeiçoar as aulas e o interesse em ajudar no entendimento dos alunos.

Vasques et al. (2021a) apontam que, apesar das aulas expositivas reforçarem a posição passiva e descontextualizada do aluno no processo de ensino-aprendizagem, esse método se faz indispensável para fornecer aos alunos o conhecimento que não podem ser obtidos por outras maneiras. Tanto que tal abordagem é comum em várias áreas da Biologia (Araújo, 2009), como o ensino de Botânica. Sendo assim, cabe ao docente balancear entre a utilização de aulas expositivas e aulas com metodologias variadas para permitir um maior aprendizado dos conteúdos.

Lima et al. (2010) citam que utilizar diversos métodos para socializar o conteúdo, possibilita um aprendizado mais efetivo no meio escolar, pois não é suficiente apenas transmitir informações, mas também relacionar o assunto de Botânica com outras ciências (Masetto, 2003). Para Kinoshita et al. (2006) a diversidade de metodologias é essencial, não apenas para o ensino de Botânica, mas para todo o processo de ensino-aprendizagem, entretanto, essas práticas podem ser prejudicadas por uma formação debilitada dos licenciados. Melo et al. (2012) relatam que despertar o interesse pela Botânica nos alunos é um desafio, principalmente se os métodos utilizados pelo professor forem restritos a livros didáticos e aulas expositivas, que não interagem com o cotidiano em que o estudante está inserido.

O uso de estratégias para despertar o interesse dos alunos para os conteúdos de Botânica é um esforço diário para os docentes (Melo et al., 2012). Nesse contexto o uso de documentários é amplamente aplicado como ferramenta para aproximar 
os alunos dos conteúdos abordados pelos docentes. Para Sousa (2020) o uso de documentários no ensino de Biologia pode estimular o sistema afetivo-avaliativo dos educandos, entretanto, a autora alerta para a importância de abordar a temática apresentada a partir de uma perspectiva crítica e analítica, evitando o seu uso como ferramentas meramente ilustrativas. Outra estratégia relatada por Melo et al. (2012) como importante ferramenta para aproximar os alunos do conteúdo de Botânica são as aulas práticas; esta incentiva os alunos a desenvolverem outras competências importantes para o estudo de Ciências no geral, como a experimentação e investigação científica, resolução de problemas, além de ajudar na compreensão de conceitos básicos. A relevância das atividades práticas no ensino de temas relacionados às ciências também é relatada por diversos autores (Krasilchik, 1987; Fernandes, 1998; Amaral, 1999; Pacheco, 2000; Possobom; Okada \& Diniz, 2003; Campos \& Oliveira, 2005; Kinoshita et al., 2006; Menezes et al., 2008; Carvalho et al., 2009).

Das estratégias citadas, merece destaque a interdisciplinaridade, que consiste em uma transversalidade do conhecimento entre diferentes disciplinas, por meio de projetos temáticos (Brasil, 2013). É notório que este método favorece os alunos, que aprendem a lidar com outras questões da vida cotidiana (Carlos, 2007). O distanciamento entre a Botânica e a realidade imediata do estudante promove desinteresse, como mencionado por Melo et al. (2012). Ao não compreender as plantas como parte do seu ambiente, nem reconhecer espécies vegetais presentes em seu cotidiano, dificilmente o aluno entenderá o valor deste conteúdo para sua vida ou para o meio ambiente (Figueiredo, Coutinho \& Amaral, 2012; Silva, Almeida Jr \& Valle, 2020).

\subsection{Sobre os estudantes}

Embora quase $60 \%$ dos discentes tenham participado da pesquisa, chama atenção à quantidade de alunos que não participaram por terem faltado (35\% do universo amostral). Este fato pode revelar alguns dos problemas crônicos da Educação Básica: a baixa frequência escolar e a evasão escolar.

No que concerne à baixa frequência esta pode ter diversos fatores, como ressalta Brasil (2020), podendo estar relacionado a aspectos de saúde, sociais, educacionais e/ou familiares. Sobre a evasão escolar, este fenômeno é amplamente discutido por estudiosos brasileiros, com destaque para Silva et al. (2019) que levantou esta questão para uma escola do Ceará. Dentre as causas que podem levar a evasão está: menor nível educacional, envolvimento em atividades antissociais de alto risco (p. ex. crime e uso de drogas), gravidez precoce (Camargo, 2006), necessidade de trabalhar, questões de saúde, motivos externos e por vontade própria, estes últimos responsabilizando parcialmente a escola por sua decisão (Silva et al., 2019). Neste contexto, a presença indispensável da comunidade externa associada à busca por mecanismos educacionais adequados a realidade dos alunos pode auxiliam na diminuição da evasão escolar (Silva et al., 2019).

Em relação ao interesse dos alunos por Botânica, todos demostraram que o assunto é notável; e a maioria (75,00 80,95\%) gosta do conteúdo, como ressaltado nas duas respostas indicadas com "sim" na Figura 1; porém, não aprofundaram na justificativa, demostrando que os alunos podem não compreender na totalidade a importância desta área. 
Figura 1. Justificativa dos alunos (em dados percentuais) para a questão do interesse em Botânica.

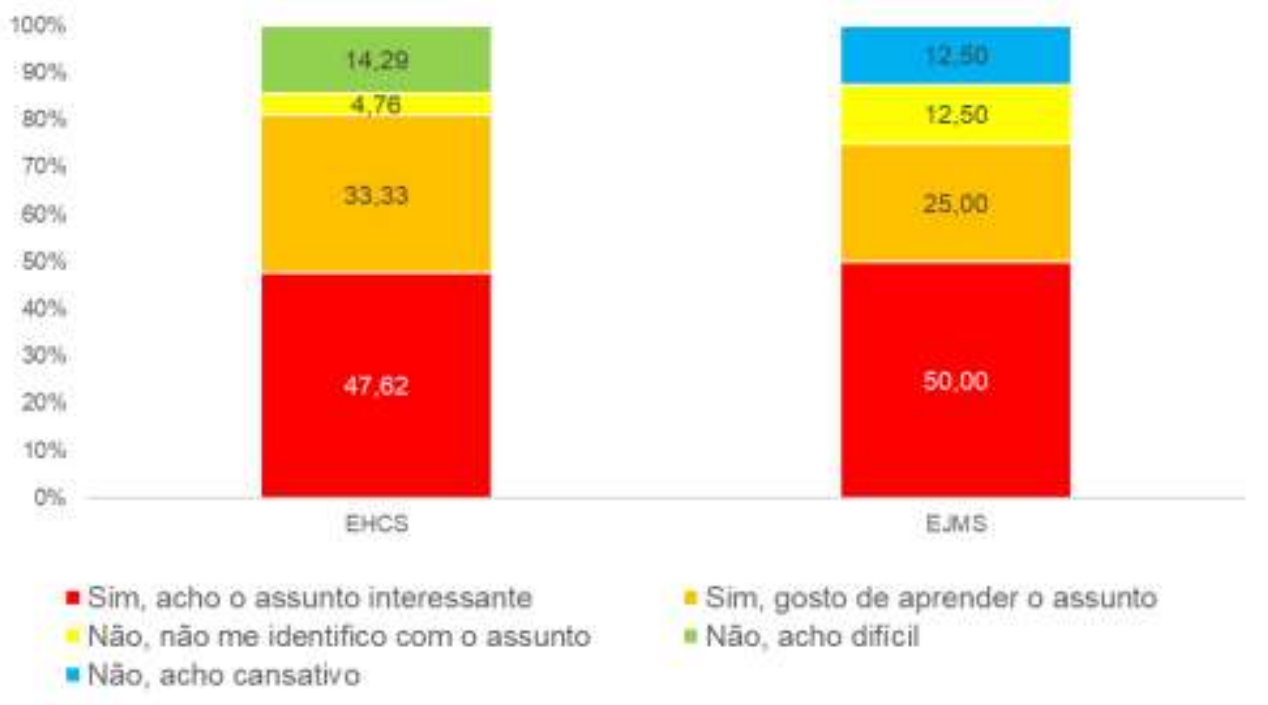

Fonte: Autores.

O interesse dos discentes pela Botânica é inferior aos dados apresentados por Pinheiro et al. (2021), porém superior aos apresentados por Batista e Araújo (2015), em ambos os trabalhos os autores relacionaram o desinteresse dos alunos pelo conteúdo ser passado de uma forma tradicional e conteudista adotada pelos professores. Pinheiro et al. (2021) adiciona que os alunos consultados consideraram o conteúdo complexo e abstrato, o que dificultava sua compreensão. Entretanto Batista e Araújo (2015) também ressaltam que a abordagem limitada e superficial como a Botânica é apresentada no ensino médio favorece o desinteresse dos alunos pelo tema.

O marcante descaso dos alunos por Botânica é recorrentemente observado e não se limita as salas de aula do Ensino Básico, mas a toda espécie humana, que avaliam preconceituosamente as plantas como seres inanimados, desinteressantes e estáticos (Salatino \& Buckeridge, 2006). Esse fenômeno é conhecido como cegueira botânica, caracterizada pela incapacidade de reconhecer a importância do papel das plantas na biosfera e no nosso cotidiano, pela dificuldade em perceber os aspectos estéticos e biológicos exclusivos das plantas e por considerar as plantas seres inferiores aos animais (Ursi et al., 2021). Essa ocorrência mostra-se como impedimento determinante na apreensão dos conteúdos (Wandersee \& Schussler, 2001; Hershey, 2002; Kinoshita et al., 2006; Ursi \& Macedo, 2016).

Em seguida foi questionado aos alunos sobre como era ministrado as temáticas referentes aos assuntos de Botânica em sala de aula. Os discentes apontaram pontos positivos para a didática do professor (Figura 2); na EHCS o destaque foi para aulas expositivas, mas ressaltando a boa explicação do docente o que facilitaria a compreensão do conteúdo; por outro lado, na EJMS os alunos ressaltaram a diversidade de metodologias (aula prática e/ou de campo, aula expositiva com slides e vídeos, confecção de herbário etc.) e atividades de pesquisa empregadas pelo professor. 
Figura 2. Diferentes abordagens utilizadas pelo professor para explicar os conteúdos de Botânica em sala de aula.

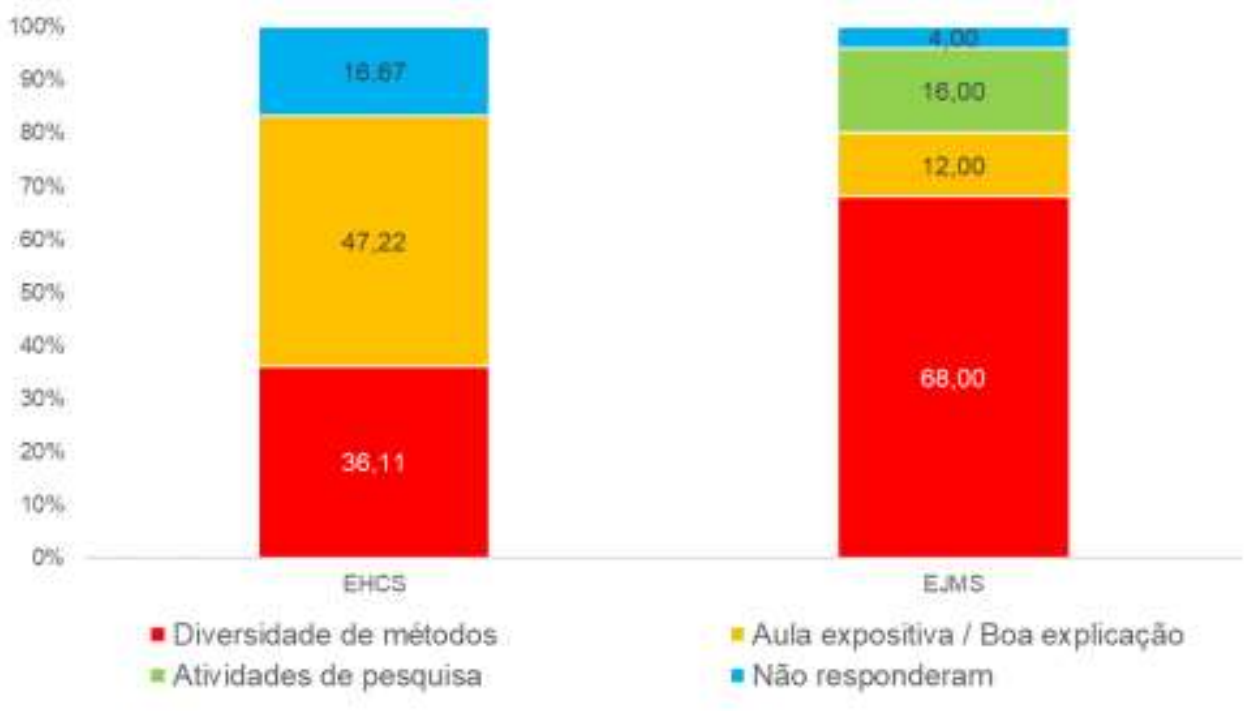

Fonte: Autores.

Como já citado anteriormente, as aulas expositivas são fundamentais. Porém, é necessário que o docente goste do assunto ministrado e procure desmitificar a Botânica de maneira racional, para que os alunos não foquem apenas em termos e conceitos, mas sim passem a entender a essência do assunto (Salatino \& Buckeridge, 2016). Neste sentido, a diversidade de metodologias (descrita pelos discentes) tem como objetivo alcançar uma educação transformadora, ao mesmo tempo em que pode facilitar o aprendizado significativo e estimular o interesse dos alunos pelos conteúdos. Também se destacam as atividades de pesquisa realizadas pelos alunos da EJMS, estas estão relacionadas a um ensino por investigação. Em tal metodologia o conteúdo torna-se mais instigante e com participação ativa do discente (Aragão, Silva \& Mendes, 2019). Assim, o uso de diferentes recursos pode ter um efeito positivamente transformador no ensino de Botânica (Katon \& Towata, 2016).

Com base nos dados das respostas anteriores em que os discentes demonstram que gostam de Botânica (Figura 1), que os conteúdos sejam ministrados de forma satisfatória (Figura 2) com uma diversidade de metodologias era de se esperar que os discentes conseguissem ver a importância da Botânica em seu cotidiano. Contudo, menos da metade dos discentes das duas escolas informaram a relevância/aplicação dos conhecimentos botânicos (e.g. medicamentos, oxigênio ou alimentação). Sendo comum não saberem (45,65\% EHCS /20,00\% EJMS) ou não apresentar importância (15,22\% EHCS /8,00\% EJMS) - Figura 3. 
Figura 3. Importância da Botânica no cotidiano dos alunos.

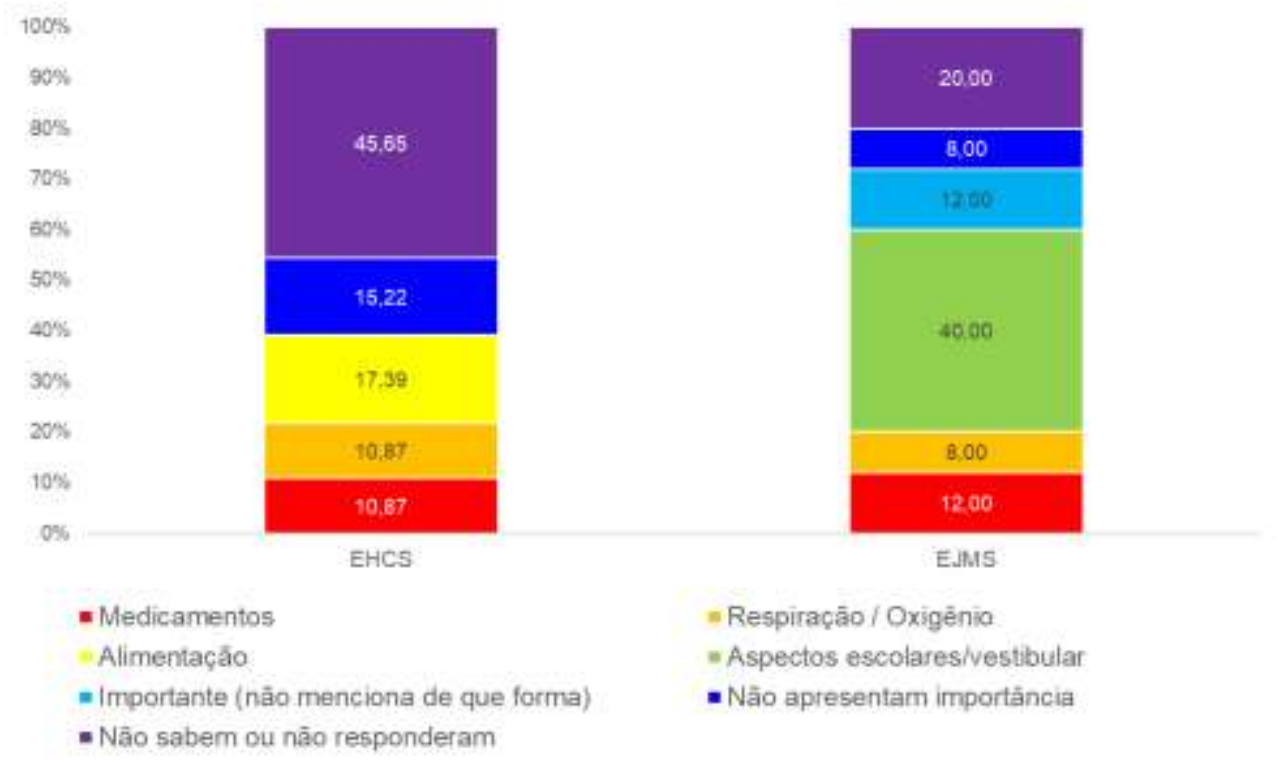

Fonte: Autores.

Outro ponto que chama a atenção é o fato de que $40 \%$ dos discentes da EJMS considera a Botânica apenas para atividades acadêmicas, e.g. atividades escolares e vestibular, não conseguindo relacionar este conteúdo ao cotidiano (Figura 3). Neste ponto, parece que a diversidade de metodologias (Figura 2) também não está sendo o suficiente para demostrar a elevada importância do conteúdo.

Em um período de dez anos (2004-2013) o ENEM, principal modo de ingresso nas universidades, teve apenas dez questões de Botânica (sete de fisiologia vegetal e três de morfologia vegetal), tendo seleções que não foi abordado em nenhuma questão; esse dado se faz importante considerando que norteiam os principais assuntos que são trabalhados no Ensino Médio (Gomes et al., 2021). A baixa presença de assuntos de Botânica no ENEM reforça o que cita Katon e Towata (2016), que ela é um tema subestimado na Biologia, o que pode gerar desinteresse e desmotivação dos estudantes. Como consequência, os discentes não conseguem perceber sua importância além dos exames de vestibular e ENEM.

Mesmo que 10\% das espécies vegetais conhecidas tenha uso imediato para a humanidade (Mancuso, 2019), isto não é contextualizado para a vida do aluno. É imprescindível que a construção do saber botânico seja feita com base no cotidiano do aluno. Katon e Towata (2016) descrevem que é mais eficiente elaborar um novo conhecimento a partir de conhecimentos prévios do que apresentar um tema totalmente independente. Utilizando o conhecimento prévio e do ambiente em que o aluno está inserido é possível fomentar a aprendizagem de maneira multidisciplinar, portanto, entendendo as plantas em seu papel nos ecossistemas, como um todo (Moreira; Feitosa \& Queiroz, 2019). Deste modo, a ausência dessa contextualização favorece a cegueira botânica e dificulta o aprendizado da área.

Segundo Ursi et al. (2021) esse fenômeno se refere a incapacidade de perceber e valorizar o Reino Vegetal, podendo ser incentivado por múltiplos fatores. Inicialmente este termo foi relacionado a questões sensorial e cognitiva, que precedem o ensino-aprendizagem (Ursi et al., 2021), relacionadas a percepção humana de que as plantas apenas compõem o cenário para a vida animal, atrelado a percepção visual do olho humano, que tende a priorizar aspectos como movimento, padrões salientes de cores, elementos conhecidos e seres ameaçadores, enquanto as plantas são percebidas como o oposto disto (Wandersee \& Schussler, 2002). 
O papel dos fatores sensório-cognitivos na teoria da cegueira botânica vem dando espaço para os aspectos culturais e de ensino, que passaram a ser vistos como fundamentais nesse fenômeno (Salatino \& Buckeridge, 2016; Ursi et al., 2021). Outro fator que interage com esse fenômeno no âmbito escolar é apresentado por Uno (2009) como "Analfabetismo Botânico", marcado pela falta de interesse e conhecimento básico da temática, deste modo retroalimentando a cegueira botânica (Ursi et al., 2018). Reunindo essas informações é importante destacar que as ações pedagógicas podem aproximar a Botânica das salas de aula, de forma mais motivadora e efetiva, visando aplacar a Cegueira e o Analfabetismo Botânicos (Neves, Bündchen \& Lisboa, 2019; Ursi et al., 2021).

Outra hipótese para essa negligência poderia ser nomeada de zoocentrismo e zoochauvinismo, que seria causado pela predileção por exemplos com animais tanto para o ensino como para mídia, como pela falta de contato com o Reino Plantae gerado pela urbanização (Hershey, 2002).

Para Silva, Cavallet e Alquini (2006), em certas ocasiões os professores alegam que articular os conteúdos ao cotidiano impediria a boa execução do programa da disciplina; porém esquecem que podem priorizar os conteúdos visando à aprendizagem significativa (Ausubel, 1966), e esta pode ser feita de forma articulada ao mesmo tempo valoriza a autonomia intelectual do discente. Assim, essas posturas dos docentes podem ter refletido nas respostas dos alunos, resultando em baixos valores para a relação entre a botânica e o cotidiano do discente (Figura 3).

Dentre as dificuldades apresentadas para entender a Botânica os estudantes destacaram principalmente a utilização de nomenclatura complexa. Essa situação é relatada por outros estudos (Ceccantini, 2006; Kinoshita et al., 2006, Nicola \& Paniz. 2016), onde os autores associam essa dificuldade dos alunos com a forma tradicional, abstrata e conteudista de transmitir o conhecimento, contudo, este modelo não estimula o desenvolvimento cognitivo daquilo que foi aprendido. No final, o aluno não é capaz de entender a lógica por trás dos termos e variações (Nunes \& Votto, 2018). A utilização de termos descontextualizados e de difícil compreensão tem como consequência a perda do entusiasmo dos estudantes, resultando na diminuição no estímulo para a aprendizagem (Macedo et al., 2012).

Assim, a dificuldade no aprendizado de botânica nas escolas de Educação Básica é frequentemente relacionada com a falta de metodologias de ensino participativas, resultando no desinteresse dos alunos pelo tema (Arruda \& Laburú, 1996; Cicillini, 2002; Ceccantini, 2006). França (2003) diz que além de apresentar a necessidade de se estudar algo, é também relevante que o aluno perceba como é possível o estudo ser agradável e fascinante.

Mesmo os alunos considerando em geral as práticas docentes satisfatórias, eles sugeriram algumas abordagens didáticas para tornar as aulas mais interessantes (Figura 4), como aulas práticas em laboratório e visitas a campo para observar as plantas em seu habitat, além de aulas mais dinâmicas e com maior aprofundamento do conteúdo. As sugestões foram mais expressivas na EHCS, curiosamente esta também foi a escola que havia mais aulas expositivas. É importante destacar o fato das aulas práticas serem citadas como sugestão nas duas escolas, mesmo o método já ser comum aplicado nas duas escolas. Os alunos alegam que gostam muito desse tipo de abordagem e que iriam apreciar caso fosse mais frequente. Segundo Gullich (2006), é possível reconhecer uma boa metodologia de ensino para o aprendizado de botânica quando o aluno é estimulado a observar o que o rodeia, como é o caso de aulas práticas, onde os alunos têm sua curiosidade aguçada favorecendo a assimilação dos conteúdos. 
Figura 4. Sugestões de abordagens metodológicas que os alunos gostariam que fossem mais frequentes.

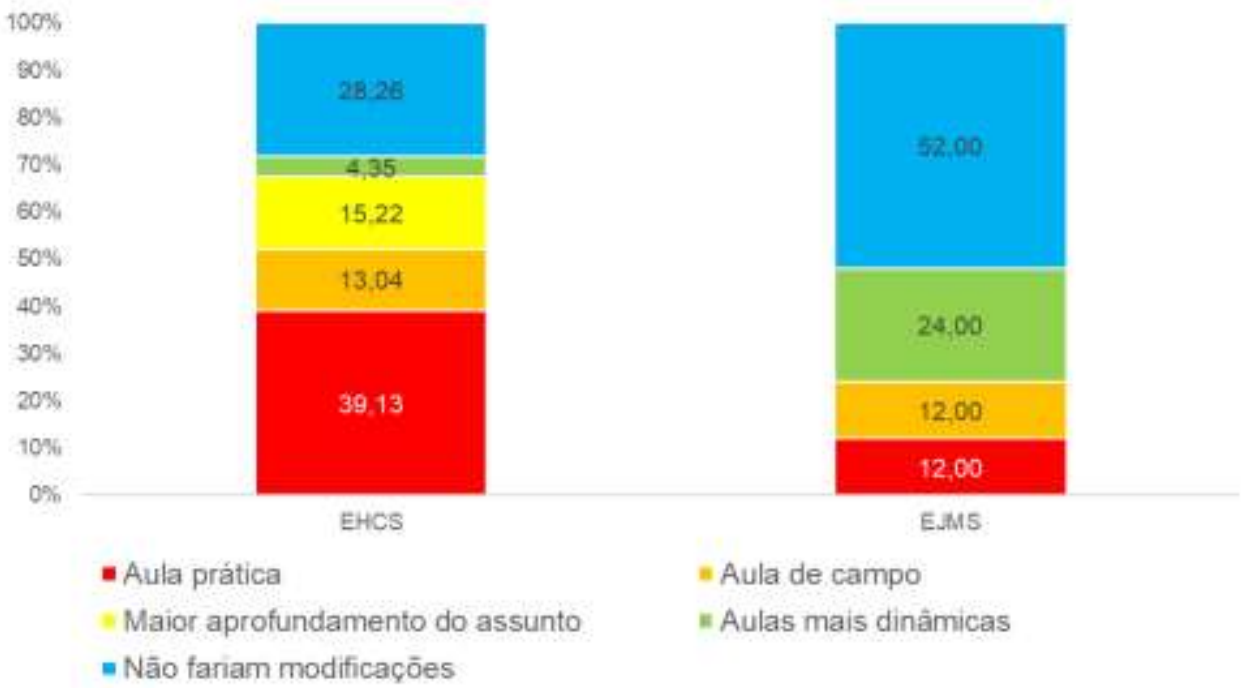

Fonte: Autores.

Outro recurso pedagógico a ser pensado, com o advento da tecnologia voltada para o ensino, é a implementação de aplicativos para smartphones. Esses elementos permitiram o acesso aos conteúdos teóricos sobre quaisquer assuntos, ajudando na superação de algumas limitações das metodologias de ensino-aprendizagem em sala de aula (Silveira, 2017). No cenário póspandemia de COVID-19, o uso das tecnologias na educação pode ser irreversível, podendo se relacionar de diversas formas com as metodologias de ensino, mas principalmente com as metodologias ativas, centradas no aluno (Schneider \& Schneider, 2020).

\section{Conclusão}

Constatou-se que a maioria dos alunos se interessa pelo assunto de Botânica e acha importante conhecê-la mais, também gostam das metodologias de ensino aplicadas pelo professor. O que demonstra que a formação acadêmica dos dois professores em Biologia, as variadas metodologias utilizadas por eles, a contextualização da disciplina de Botânica com outras ciências e suas atuações são fundamentais nesse processo, pois contribui para estimular o conhecimento dos alunos e fazê-los se interessar pela disciplina. Fazendo-se necessário que o professor busque estratégias e metodologias que propiciem um ensino estimulante e que resultem em uma educação científica de qualidade, que aproxime a Botânica da realidade do aluno.

Contudo, mesmo com a diversidade de metodologias, em especial atividades práticas e de pesquisa que permite um maior dinamismo e motivação no aprendizado, muitos alunos ainda não conseguem perceber a botânica em seu cotidiano ou que deve ser utilizada apenas para os exames (provas escolares, vestibular e ENEM). Possivelmente isso ocorra devido a dificuldade da nomenclatura e à cegueira botânica.

Diante desse panorama, é interessante desenvolver novos estudos em diferentes frentes, como: (i) entender quais as dificuldades que os professores apresentam para ensinar Botânica, especialmente à questão nomenclatural; (ii) munir e capacitar os professores da rede básica para proporcionarem essas atividades para os seus alunos; (iii) avaliar a receptividade, por parte dos alunos, a diferentes metodologias ativas no ensino de Botânica.

\section{Referências}

Amaral, I. A. (1999). Currículo de ciências: das tendências clássicas aos movimentos atuais de renovação. In: Barreto, E. S. S. (Ed.). Os currículos do ensino fundamental para as escolas públicas brasileiras: Autores Associados, 200-232. 
Aragão, A. A. S., Silva, J. J. J., \& Mendes, S. M. (2019). Ensino de ciências por investigação: o aluno como protagonista do conhecimento. Revista Vivências em Ensino de Ciências, 4(3), 75-84.

Araújo, E. S. N. N. (2009). Ensino de biologia em espaços não formais. In: Caldeira, A. M. A. (Ed.). Introdução à didática da biologia: educação para ciências, (10a. ed.): Escrituras Editora.

Arruda, S. M., \& Laburú, C. E. (1996). Considerações sobre função do experimento no ensino de ciências. Pesquisa em Ensino de Ciências e Matemática, 5 , 14-24.

Ausubel, D. P. (1966). Cognitive structure and the facilitation of meaningful verbal learning. In: Anderson, R. C., \& Ausubel, D. P. (Ed.) Readings in the psychology of cognition: Holt, Rinehart and Winston, p. 98-112.

Batista, L. N., \& Araújo, J. N. (2015). A botânica sob o olhar dos alunos do ensino médio. Revista Areté, 8(15), 109-120.

Batista Júnior, J. R., Sato, D. T. B., \& Melo, I. F. (2018). Análise de discurso crítica: para linguistas e não linguistas: Parábola, 224 p.

Brasil-Peixoto, S. N. R., Carneiro Júnior, G. R., Morais, C. R. S., Mendes, R. M. S., \& Edson-Chaves, B. (2021). Criação de um herbário virtual como recurso didático para ensino de botânica. Research, Society and Development, 10(1) http://dx.doi.org/10.33448/rsd-v10i1.11920

Brasil. (2006a). Ministério da Educação. Secretaria de Educação Básica. Biologia: Ensino Médio. (Col. Explorando o ensino). <http://portal.mec.gov.br/index.php?option=com_content\&view=article \&id=12314\&Itemid=632>.

Brasil. (2006b). Ministério da Educação. Secretaria de Educação Básica. Orientação curricular para o Ensino Médio. MEC/SEB. Cap.01, p.15-51.

Brasil.(2013). Ministério da Educação. Secretaria de Educação Básica. Diretrizes Curriculares Nacionais Gerais da Educação Básica: MEC, SEB, DICEI, p. 27-29.

Brasil. (2020). Ministério da Cidadania. Secretaria Especial de Desenvolvimento Social. Secretaria Nacional de Renda de Cidadania. Guia para acompanhamento das condicionalidades do Programa Bolsa Família. <http://www.mds.gov.br/ webarquivos/publicacao/ bolsa_familia/ Guias_Manuais/ Acompanhamento_condicionalidades.pdf>.

Camargo, J. M. (2006). Dívida por educação: efeitos sobre o crescimento e pobreza. <http://www.unesdoc.unesco.org>.

Camargo-Oliveira, R. (2007). Iniciativas para o aprimoramento do ensino de botânica. In: Barbosa, L. M., \& Santos Júnior, N. A. (Orgs.). A botânica no Brasil: pesquisa, ensino e políticas públicas ambientais. São Paulo: Sociedade Botânica do Brasil, p. 511-515.

Campos, A. J. M., \& Oliveira, M. R. (2005). Práticas de campo como ferramenta didática no ensino de ecologia no ensino médio. Trabalho de Conclusão de Curso (Formação continuada em Ciências da Natureza, Matemática e suas Tecnologias) - Universidade Federal do Rio de Janeiro, Rio de Janeiro.

Carlos, J. G. (2007). Interdisciplinaridade no ensino médio: desafios e potencialidades. Dissertação (Mestrado em Ensino de Ciências) - Universidade de Brasília, Brasília.

Carvalho, A. M. P., Azevedo, M. C. P. S., Nascimento, V. B., Cappechini, M. C. M., Vannuchi, A. I., Castro, R. S., Pietrocola, M., Vianna, D. M., \& Araújo, E. R. S. (2009). Ensino de ciências: unindo a pesquisa e a prática: Cengage Learning.

Ceccantini, G. (2006). Os tecidos vegetais têm três dimensões. Revista Brasileira de Botânica, 29(2), 335-337.

Chassot, A. I. Alfabetização científica: questões e desafios para a educação. (3a ed.): Unijuí. 436 p.

Cicillini, G. A. (2002). Conhecimento ciêntifico e conhecimento escolar: aproximações e distanciamentos. In: Cicillini, G. A., \& Nogueira, S. V. (Org.) Educação escolar: políticas, saberes e práticas pedagógicas. Uberlândia: Edufu, p. 37-84.

Costa, E. A., Duarte, R. A. F., \& Gama, J. A. S. (2019). A gamificação da botânica: uma estratégia para a cura da "cegueira botânica". Revista Insignare Scientia, 2(4), 79-99.

Dias, F. Y. E. C., Oliveira, R. D., Mendes, R. M. S., Pantoja, L. D. M., Bonilla, O. H., \& Edson-Chaves, B. (2020). O papel da Feira de Ciências como estratégia motivadora para ensino de botânica na educação básica. Hoehnea, 47: 1-12. http://dx.doi.org/10.1590/2236-8906-55/2019.

Edson-Chaves, B., Oliveira, R. D., Chikowski, R. S., Mendes, R. M. S., \& Medeiros, J. B. L. P. (2015). Ludo vegetal: uma nova alternativa para a aprendizagem de botânica. Revista Brasileira de Biociências, 13(3), 194-200.

Fernandes, H. L. (1998). Um naturalista na sala de aula. Ciência \& Ensino, 1(5), 11-12.

Ferreira; M. M. S., Furlan, C. M., \& Motta, L. B. (2012). A importância das plantas. In: Furlan, C. M.; Santos, D. Y. A. C., \& Chow, F. A botânica do cotidiano: Holos, $240 \mathrm{p}$.

Ferretti, C. J. (2016). Reformulações do Ensino Médio. Holos, 32(6), 71-91.

Figueiredo, J. A., Coutinho, F. A., \& Amaral, F. C. (2012). O ensino de botânica em uma abordagem ciência, tecnologia e sociedade. In: Seminário hispano brasileiro CTS, 2., São Paulo, 2012. Anais... <http://revistapos.cruzeirodosul.edu.br/index.php/rencima/article/viewFile/420/353>.

França, C. (2003). A falta de vontade para estudar. 〈http://www.eaprender.com.br/tiki-smartpages_view. php?pageld=1113>.,

Gil, A. C. (1999). Métodos e técnicas de pesquisa social. (5a ed.): Atlas, 206 p.

Gil, A. C. (2002). Como elaborar projetos de pesquisa. (4a ed.): Atlas, 176 p. 
Gomes, N. F., Morais, C. R. S., Carneiro Júnior, G. R., Lucena, E. M. P., Edson-Chaves, B., \& Mendes, R. M. S. (2021). A botânica nos vestibulares da UECE e do ENEM de 2004-2013. In: Edson-Chaves, B.; Mendes, R. M. S., Bonilla, O. E., \& Lucena, E. M. P. (Org.). Contextualizando o ensino de botânica e ecologia. Cap. 3, p.26-6.

Gullich, R. I. C. (2006). As práticas de ensino de botânica e a SBB. In: Congresso Nacional de Botânica, 57., 2006, Porto Alegre. Anais: Sociedade de Botânica do Brasil. p. 756.

Hershey, D. R. (1996). A historical perspective on problems in botany teaching. The American Biology Teacher, 58(6), 340-347.

Hershey, D. (2002). Plant blindness: we have met the enemy and he is us. Plant Science Bulletin, 48, 78-84.

Katon, G. F., \& Towata, N. (2016). Por que a botânica é tão chata? In: Silva, F. N., Raimundo, S. G., Saito, L. C., Navarro, B. V., Hidalgo, E. M. P., Moreira, F. A., Gagliano, J., Carvalho, W. R. S., Coutinho Neto, A. A., Dias, D. L. O., \& Furlan, C. M. (Org.). VI Botânica no Inverno: Instituto de Biociências da Universidade de São Paulo, Departamento de Botânica, p. 86-91.

Kinoshita, L. S., Torres, R. B., Tamashiro, R. B., \& Forni-Martins, E. R. (2006). A botânica no ensino básico: relatos de uma experiência transformadora. São Carlos: RiMa.143 p.

Krasilchik, M. (1987). O professor e o currículo das ciências: EPU/EDUSP, 50 p.

Lemos, V. D. O. T., Lucena, E. M. P., Bonilla, O. H., Mendes, R. M. S., \& Edson-Chaves, B. (2018). Paródias como facilitador no processo ensino-aprendizagem de anatomia vegetal no ensino superior. Revista Brasileira de Biociências, 16(2), 53-61.

Lima, R. M. S., Lima, A. N., Silva, R. V., Silva, V. H., \& Araújo, M. L. F. (2010). Ensino de biologia em escolas públicas estaduais: um olhar a partir das modalidades didáticas. In: Jornada de ensino, pesquisa e extensão - JEPEX, 10., 2010, Recife. Anais: UFRPE.

Macedo, M., Katon, G. F., Towata, N., \& Ursi, S. (2012). Concepções de professores de biologia do ensino médio sobre o ensino-aprendizagem de botânica. In Encontro Ibero-americano sobre investigação em ensino de ciências, 4., 2012, Porto Alegre. Anais: UFRGS, p. 387-401.

Machado, C. C., \& Amaral, M. B. (2015). Memórias ilustradas: aproximações entre formação docente, imagens e personagens botânicos. Alexandria, Revista de Educação em Ciência e Tecnologia, 8(2), 7-20.

Mancuso, S. (2019). Revolução das plantas: Ubu. 192p.

Marinho, L. C., \& Valle, M. G. (2020). Práticas em sistemática vegetal em tempos de pandemia: construindo um Herbal em casa. Botânica Pública, 1(1), 2125 .

Masetto, M. T. (2003). Competência pedagógica do professor universitário. Summus. 194 p.

Melo, E. A., Abreu F. F., Andrade, A. B., \& Araújo, M. I. O. (2012). A aprendizagem de botânica no ensino fundamental: dificuldades e desafios. Scientia Plena, $8(10), 1-8$.

Menezes, L. C. M., Souza, V. C., Nicomedes, M. P., Silva, N. A., Quirino, M. R., Oliveira, A. G., Andrade, R. R. D., \& Santos, B.A.C. (2008). Iniciativas para o aprendizado de botânica no ensino médio. In: Encontro de iniciação à docência, 11, 2008, João Pessoa. Anais: UFPB.

Minhoto, M. J. (2002). Breve histórico sobre botânica. <http://www.botanicasp.org.br/educacao/historico.htm>.

Moreira, L. H. L., Feitosa, A. A. F. M. A., \& Queiroz, R. T. (2019). Estratégias pedagógicas para o ensino de botânica na educação básica. Experiências em Ensino de Ciências, 14(2), 368 - 384

Nascimento, B. M., Donato, A. M., Siqueira, A. E., Barroso, C. B., Souza, A. C. T., Lacerda, S. M., \& Borim. D. C. D. E. (2017). Propostas pedagógicas para ensino de botânica nas aulas de ciências: diminuindo entraves. Revista Electrónica de Enseñanza de las Ciencias, 16(2), $298-315$.

Neves, A., Bündchen, M., \& Lisboa, C. P. (2019). Cegueira botânica: é possível superá-la a partir da educação? Ciênc. Educ., Bauru, 25(3), 745-762.

Nicola, J. A., \& Paniz, C. M. (2016). A importância da utilização de diferentes recursos didáticos no ensino de biologia. Revista NEaD-Unesp, 2(1), 355-381.

Nunes, M. R., \& Votto, A. P. S. (2018). A etimologia como ferramenta para a aprendizagem significativa de biologia. Revista Thema, 15(2), 592-602.

Pacheco, D. A. (2000). Experimentação no ensino de ciências. Ciência \& Ensino, 10(2), 7.

Paz, I. N., \& Souza, J. M. (2016). Utilização de história em quadrinhos como ferramenta de avaliação no processo de ensino- aprendizagem de botânica no Clube de Ciências, Boletim do Museu Integrado de Roraima, 10(1), 10-19.

Pinheiro, L. F., Bonilla, O. H., Mendes, R. M. S., \& Lucena, E. M. P. (2021) Percepção dos alunos de ensino médio sobre a botânica. In: Edson-Chaves, B., Mendes, R. M. S., Bonilla, O. E., \& Lucena, E. M. P. (Org.). Contextualizando o ensino de botânica e ecologia. Cap. 4, p.37-52.

Possobom, C. C. F., \& Okada, F. K. \& Diniz, R. E. S. (2003). As atividades práticas de laboratório no ensino de biologia e ciências: relato de uma experiência. Botucatu: UNESP.

Pes, C. D. S. (2019). Conhecimento pedagógico do conteúdo em botânica: desafios para a formação na educação básica. Tese (Doutorado em Ensino de Ciências e Matemática). Programa de Pós-Graduação em Ensino de Ciências e Matemática - Universidade Franciscana, Santa Maria.

Salatino, A., \& Buckeridge, M. (2016). Mas de que te serve saber botânica? Estudos Avançados, 30(87), 177-196. 
Research, Society and Development, v. 10, n. 4, e18110413660, 2021

(CC BY 4.0) | ISSN 2525-3409 | DOI: http://dx.doi.org/10.33448/rsd-v10i4.13660

Silva, J. R. S., Guimarães, F., \& Sano, P. T. (2016). Teaching of botany in higher education: representations and discussions of undergraduate students. Revista Eletronica de Enseñanza de las Ciencias, 15(3), 380-393.

Schneider, E. I., \& Schneider, A. B. (2020). Educação em tempos de pandemia. In: Machado, D. P. Educação em tempos de covid-19: reflexões e narrativas de pais e professores: Dialética e Realidade. p. 51-64.

Silva, A. N. F., Almeida Júnior, E. B., \& Valle, M. G. (2020). Exsicatas como recurso didático: contribuições para o ensino de botânica/exsicate as didactic resource: support for the teaching of botany. Brazilian Journal of Development, 6(5), 24632-39.

Silva, F. E. M., Silva, S. A., Juca, S. C. S, Monteiro, A. O., Lemos, P. B. S., \& Rocha, P. C. S. (2019). Evasão escolar como desafio contemporâneo à Escola de Ensino Médio Luiz Girão, em Maranguape-CE. Research, Society and Development, 8(8), e43881251.

Silva. J. J. L., Cavalvante. F. L. P., Xavier, V. F., \& Gouveia, L. F. P. (2019). Produção de exsicatas como auxílio para o ensino de botânica na escola. Conexões Ciência e Tecnologia. 13(1), 30-37. 10.21439/conexoes.v13i1.1488

Silva, L. M., Cavallet, V. J., \& Alquini, Y. (2006). O professor, o aluno e o conteúdo no ensino de botânica. Revista Educação, 31(1), 67-80.

Silveira, A. C. M. (2017). As tecnologias da informação e comunicação no ensino de ciências: desenvolvimento de um aplicativo mobile de histologia animal e sua aplicação em sala de aula. Trabalho de Conclusão de Curso (Graduação em Ciências Biológicas), Instituto de Biologia Roberto Alcântara Gomes, Universidade do Estado do Rio de Janeiro, Rio de Janeiro.

Sousa, J. C. (2020). Documentários científicos sobre o mundo natural no ensino de biologia. Ciência \& Educação, 26, 1-18.

Uno, G. E. (2009). Botanical literacy: what and how should students learn about plants? American Journal of Botany, 96(10), 1753-1759.

Ursi, S., \& Macedo, M. (2016). Botânica na escola: uma proposta para o ensino de histologia vegetal. Revista da SBEnBio, 9, 2723-33.

Ursi, S., Barbosa, P. P., Sano, P. T., \& Berchez, F. A. S. (2018). Ensino de botânica: conhecimento e encantamento na educação científica. Estudos Avançados, 32(94).

Ursi, S., Freitas, K. C., Vasques, D. T. (2021). Cegueira Botânica e sua mitigação: um objetivo central para o processo de ensino-aprendizagem de Biologia. In: Vasques, D. T., Freitas, K. C., \& Ursi, S. (Ed.) Aprendizado ativo no ensino de botânica. São Paulo: Instituto de Biociências, Universidade de São Paulo, Cap. 2, p. $12-30$.

Vasques, D. T., Freitas, K. C., \& Ursi, S. (2021a). Aprendizado ativo: conceito, história e práticas. In: Vasques, D. T.; Freitas, K. C. \& Ursi, S. (Ed.) Aprendizado ativo no ensino de botânica: Instituto de Biociências, Universidade de São Paulo, Cap. 4, p. 52-82.

Vasques, D. T., Freitas, K. C., \& Ursi, S. (2021b). Aprendizado ativo no ensino de botânica: Instituto de Biociências, Universidade de São Paulo.

Wandersee, J. H., \& Schussler, E. E. (2001). Towards a theory of plant blindness. Plant Science Bulletin, 47(1), 2-9. 